\title{
Polyakov Loop Fluctuations in Terms of Dirac Eigenmodes
}

\section{$\operatorname{AUTHOR}(\mathrm{S}):$}

Doi, Takahiro M.; Redlich, Krzysztof; Sasaki, Chihiro; Suganuma, Hideo

\section{CITATION:}

Doi, Takahiro M....[et al]. Polyakov Loop Fluctuations in Terms of Dirac Eigenmodes. Acta Physica Polonica B Proceedings Supplement 2017, 10(3): 543-547

\section{ISSUE DATE:}

2017

URL:

http://hdl.handle.net/2433/229440

\section{RIGHT:}

The intellectual rights fully remain at the author or his/her employer. They also retain the right to copy, distribute and display the published version of this Article, and to create derivative works, as long as they credit the original Article source. Articles are published under the conditions of Creative Common License, CC-BY 4.0. 


\title{
POLYAKOV LOOP FLUCTUATIONS IN TERMS OF DIRAC EIGENMODES*
}

\author{
Takahiro M. Doi ${ }^{\mathrm{a}}$, Krzysztof Redlich ${ }^{\mathrm{b}}$, Chiniro Sasaki $^{\mathrm{b}}$ \\ Hideo Suganuma ${ }^{a}$

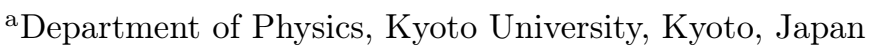 \\ ${ }^{b}$ Institute of Theoretical Physics, University of Wrocław, Wrocław, Poland
}

\section{(Received February 16, 2017)}

We investigate, both numerically and analytically, the Polyakov loop and its fluctuations in terms of Dirac eigenmodes in the lattice QCD. Based on the analytical relation between the Polyakov loop and the Dirac eigenmodes, it is found that the Polyakov loop and its fluctuations, contrary to the chiral condensate, are insensitive to the density of the low-lying Dirac modes. This result suggests that there is no direct one-to-one correspondence between quark confinement and chiral symmetry breaking in QCD. Our argument is also generalized for the Wilson-type Dirac operator on the lattice.

DOI:10.5506/APhysPolBSupp.10.543

\section{Introduction}

Quark confinement and chiral symmetry breaking are important nonperturbative phenomena to understand the rich properties of the QCD vacuum. In spite of their importance, these phenomena and their relations are still not sufficiently understood.

In many lattice-QCD calculations at finite temperature both quark deconfinement and chiral restoration occur in the same temperature range [1,2]. However, this result does not necessarily imply that quark confinement and chiral symmetry breaking are strongly correlated, as there are some opposite findings. For example, in Ref. [3], it was shown that for physical values of quark masses, the deconfinement crossover temperature is significantly higher than the chiral crossover temperature. In addition, even after removing the low-lying Dirac modes, "hadrons" as bound state of quarks and gluons can be still observed on the lattice [4], and the Polyakov loop and the string tension are almost unchanged [5].

* Presented at the "Critical Point and Onset of Deconfinement" Conference, Wrocław, Poland, May 30-June 4, 2016. 
In this paper, we present the analytic relation between the Polyakov loop fluctuations and Dirac eigenmodes, and show that the low-lying Dirac modes have negligible contribution to the Polyakov loop fluctuations. Finally, as a recent progress, we generalize our argument for the Wilson-type Dirac operator on the lattice in order to remove the fermionic doubler modes.

\section{Polyakov loop fluctuations and Dirac eigenmode}

In this section, we introduce the Polyakov loop fluctuations [6] and the Dirac eigenmodes on the lattice [5].

We consider the $\mathrm{SU}\left(N_{\mathrm{c}}\right)$ lattice QCD on a standard square lattice with spacing $a$, and the notation of sites $s=\left(s_{1}, s_{2}, s_{3}, s_{4}\right)\left(s_{\mu}=1,2, \cdots, N_{\mu}\right)$. The link-variables $U_{\mu}(s)=e^{i a g A_{\mu}(s)}$ are expressed by gauge fields $A_{\mu}(s) \in$ $\mathrm{SU}\left(N_{\mathrm{c}}\right)$ and gauge coupling $g$. The lattice is taken to be spatially isotropic, i.e., $N_{1}=N_{2}=N_{3} \equiv N_{\sigma}$ and $N_{4} \equiv N_{\tau}$, with $N_{\sigma} \leq N_{\tau}$. All $\gamma$-matrices are defined to be hermitian, thus $\gamma_{\mu}^{\dagger}=\gamma_{\mu}$.

We define the link-variable operator $\hat{U}_{ \pm \mu}$ by the matrix element as

$$
\left\langle s\left|\hat{U}_{ \pm \mu}\right| s^{\prime}\right\rangle=U_{ \pm \mu}(s) \delta_{s \pm \hat{\mu}, s^{\prime}} \quad \text { with } \quad\left\langle s, N_{t}\left|\hat{U}_{4}\right| s, 1\right\rangle=-U_{4}\left(s, N_{t}\right) .
$$

The temporal anti-periodicity of the link-variable operator is imposed for the temporal anti-periodicity of the Dirac operator at finite temperature. Using the link-variable operators, the Polyakov loop $L$ and the $Z_{3}$ rotated Polyakov loop $\tilde{L}$ are defined as

$$
L \equiv-\frac{1}{N_{\mathrm{c}} V} \operatorname{Tr}_{\mathrm{c}}\left\{\hat{U}_{4}^{N_{\tau}}\right\}, \quad \tilde{L} \equiv L e^{2 \pi k i / 3}, \quad(k=0, \pm 1),
$$

where $V$ is the four dimensional volume of the lattice and $k$ is taken so that $\tilde{L}$ belongs to the real sector. Then, the three types of the Polyakov loop susceptibilities are introduced

$$
T^{3} \chi_{Y}=\frac{N_{\sigma}^{3}}{N_{\tau}^{3}}\left[\left\langle Y^{2}\right\rangle-\langle Y\rangle^{2}\right], \quad\left(Y=|L|, L_{\mathrm{L}}, L_{\mathrm{T}}\right)
$$

where $L_{\mathrm{L}} \equiv \operatorname{Re}(\tilde{L}), L_{\mathrm{T}} \equiv \operatorname{Im}(\tilde{L})$, and $\langle x\rangle$ denotes an average over all gauge configurations.

We introduce two ratios of the Polyakov loop susceptilitities, $R_{\mathrm{A}} \equiv \frac{\chi_{\mathrm{A}}}{\chi_{\mathrm{L}}}$ and $R_{\mathrm{T}} \equiv \frac{\chi_{\mathrm{T}}}{\chi_{\mathrm{L}}}$. These ratios are superior to the Polyakov loop itself in their properties for the renormalization, because the ambiguities from the renormalization scheme can be avoided. Also, from the lattice QCD calculations, it is found that the ratio $R_{\mathrm{A}}$ is very sensitive probe for the quark deconfinement [6]. 
Using the link-variable operators, the eigenvalue equation of the naive Dirac operator $\hat{D}$ on the lattice is expressed as

$$
\hat{\not D}|n\rangle=i \lambda_{n}|n\rangle, \quad \hat{D}=\frac{1}{2 a} \sum_{\mu=1}^{4} \gamma_{\mu}\left(\hat{U}_{\mu}-\hat{U}_{-\mu}\right) .
$$

The Dirac eigenvalue $i \lambda_{n}$ is purely imaginary because $\hat{D}$ is anti-hermitian. These Dirac eigenstates $|n\rangle$ have the completeness of $\sum_{n}|n\rangle\langle n|=1$. The low-lying modes of the Dirac eigenmodes are important modes for chiral symmetry breaking because they have the dominant contribution to the chiral condensate $\langle\bar{\psi} \psi\rangle$ known as the Banks-Casher relation [7].

\section{Polyakov loop fluctuations in terms of Dirac eigenmode}

In the following, we introduce the analytical relations connecting the Polyakov loop fluctuations and the Dirac eigenmodes on the lattice. We then discuss the relation between the chiral symmetry breaking and quark confinement.

The analytical relation between the Polyakov loop and the Dirac eigenmodes in lattice QCD was derived in Refs. [8] and [9]. In particular, on the temporally odd-number lattice, where the temporal length of the lattice is odd number, the above relation is rather transparent and reads

$$
L=-\frac{(2 a i)^{N_{\tau}-1}}{12 V} \sum_{n} \lambda_{n}^{N_{\tau}-1}\left\langle n\left|\hat{U}_{4}\right| n\right\rangle .
$$

The Dirac matrix element of the link-variable operator $\left\langle n\left|\hat{U}_{4}\right| n\right\rangle$ is finite, thus $\left|\left\langle n\left|\hat{U}_{4}\right| n\right\rangle\right|<1$. From Eq. (5) and the definitions of the Polyakov loop fluctuations, one can also express different Polyakov loop susceptibilities by the Dirac eigenmodes [10]. In particular, the important quantity $R_{\mathrm{A}}$ can be expressed in terms of the Dirac modes as

$$
R_{\mathrm{A}}=\frac{\left\langle\left|\sum \lambda_{n}^{N_{\tau}-1} \hat{U}_{4}^{n n}\right|^{2}\right\rangle-\left\langle\left|\sum \lambda_{n}^{N_{\tau}-1} \hat{U}_{4}^{n n}\right|\right\rangle^{2}}{\left\langle\left(\sum \lambda_{n}^{N_{\tau}-1} \operatorname{Re}\left(e^{2 \pi k i / 3} \hat{U}_{4}^{n n}\right)\right)^{2}\right\rangle-\left\langle\sum \lambda_{n}^{N_{\tau}-1} \operatorname{Re}\left(e^{2 \pi k i / 3} \hat{U}_{4}^{n n}\right)\right\rangle^{2}},
$$

where we have defined $\hat{U}_{4}^{n n}=\left\langle n\left|\hat{U}_{4}\right| n\right\rangle$, and the sum is taken over $n$.

From Eq. (6), it is clear that due to the damping factors $\lambda_{n}^{N_{\tau}-1}$, the low-lying Dirac modes have relatively small contribution to each sum $\sum_{n}$ over Dirac modes. Consequently, the ratio $R_{\mathrm{A}}$ should be insensitive to the 
density of the low-lying Dirac modes. Indeed, this analytical observation indicating that there is no direct one-to-one correspondence between quark confinement and chiral symmetry breaking in QCD was confirmed within the numerical calculations in lattice QCD [10].

\section{Removal of the doubler modes}

In this section, we improve the above results by removing the fermionic doubler modes on the lattice. In fact, the naive Dirac operator (4) includes the unphysical modes which are referred to as fermionic doubler modes [11].

An easy procedure for removing the doubler modes is to use the WilsonDirac operator defined as

$$
\hat{D}_{\mathrm{W}}=4 r / a-\frac{1}{2 a} \sum_{\mu=1}^{4}\left[\left(r-\gamma_{\mu}\right) \hat{U}_{\mu}+\left(r+\gamma_{\mu}\right) \hat{U}_{-\mu}\right],
$$

where $r$ is the Wilson parameter. The eigenvalue equation of the WilsonDirac operator is written as

$$
\hat{D}_{\mathrm{W}}|n\rangle_{\mathrm{W}}=\Lambda_{n}|n\rangle_{\mathrm{W}}, \quad \Lambda_{n} \in \boldsymbol{C} .
$$

We consider the temporally odd-number lattice and we set the temporal size as $N_{\tau}=2 N+1$. The derivation of the relation between the Polyakov loop and the Wilson-Dirac modes is essentially the same as the derivation of Eq. (5) [9]. The actual form of the relation is expressed as

$$
L=\frac{2 a^{N}}{3 V} \sum_{n}\left(\Lambda_{n}\right)^{N}\left\langle n\left|\hat{U}_{4}^{N+1}\right| n\right\rangle_{\mathrm{W}},
$$

where we set $r=1$ and abbreviate the bra-vector ${ }_{\mathrm{w}}\langle n|$ to $\langle n|$. This analytical relation connects the Polyakov loop and the Wison-Dirac modes. Thus, it is confirmed that the low-lying Wilson-Dirac modes with $\left|\Lambda_{n}\right| \sim 0$ also have negligible contribution to the Polyakov loop, and then its fluctuations.

From the viewpoint of the chiral symmetry on the lattice, the overlapDirac operator is better than the Wilson-Dirac operator [12]. An attempt to discuss the relation between the Polyakov loop and the overlap-Dirac modes is in progress.

\section{Conclusion}

We have derived a set of the analytical relations connecting the Polyakov loop fluctuations and the Dirac eigenmodes for the naive discretization and the Wilson-type of the Dirac operator. These analytical relations show that 
the low-lying Dirac modes have little contribution to the Polyakov loop fluctuations. Our result suggests that there is no one-to-one correspondence between quark confinement and chiral symmetry breaking in QCD.

T.M.D. and H.S. are supported by the Grant-in-Aid for JSPS Fellows (grant No. 15J02108) and the Grants-in-Aid for Scientific Research (grant No. 15K05076) from Japan Society for the Promotion of Science. The work of K.R. and C.S. has been partly supported by the National Science Centre, Poland (NCN) under Maestro grant No. DEC-2013/10/A/ST2/0010. The lattice QCD calculations were performed on NEC-SX8R and NEC-SX9 at the Osaka University.

\section{REFERENCES}

[1] F. Karsch, Lect. Notes Phys. 583, 209 (2002) and references therein.

[2] A. Bazavov et al., Phys. Rev. D 85, 054503 (2012).

[3] Y. Aoki et al., Nature (London) 443, 675 (2006).

[4] C.B. Lang, M. Schrock, Phys. Rev. D 84, 087704 (2011); L.Ya. Glozman, C.B. Lang, M. Schrock, Phys. Rev. D 86, 014507 (2012).

[5] S. Gongyo, T. Iritani, H. Suganuma, Phys. Rev. D 86, 034510 (2012);

T. Iritani, H. Suganuma, Prog. Theor. Exp. Phys. 3, 033 B03 (2014).

[6] P.M. Lo et al., Phys. Rev. D 88, 014506 (2013); 88, 074502 (2013).

[7] T. Banks, A. Casher, Nucl. Phys. B 169, 103 (1980).

[8] H. Suganuma, T.M. Doi, T. Iritani, Prog. Theor. Exp. Phys. 2016, $013 \mathrm{~B} 06$ (2016).

[9] T.M. Doi, H. Suganuma, T. Iritani, Phys. Rev. D 90, 094505 (2014).

[10] T.M. Doi, K. Redlich, C. Sasaki, H. Suganuma, Phys. Rev. D 92, 094004 (2015).

[11] H.J. Rothe, Lattice Gauge Theories, World Scientific, 2012.

[12] H. Neuberger, Phys. Lett. B 417, 141 (1998). 